EPJ Web of Conferences 41, 01003 (2013)

DOI: $10.1051 /$ epjconf/20134101003

(C) Owned by the authors, published by EDP Sciences, 2013

\title{
Control of High Harmonic Generation using an off-axis beam
}

\author{
K. B. Dinh, P. Hannaford and L. V. Dao \\ $A R C$ Centre of Excellence for Coherent X-Ray Science and Centre for Atom Optics and Ultrafast \\ Spectroscopy, Swinburne University of Technology, Hawthorn, Victoria 3122, Australia
}

\begin{abstract}
An off-axis beam is used to control the phase matching condition in the high harmonic generation process in a semi-infinite gas cell leading to suppression or enhancement of the harmonic emission.
\end{abstract}

\section{Introduction}

High order harmonic generation (HHG) that occurs in the interaction between an intense laser pulse and a gas medium can provide a table-top coherent radiation source in the short wavelength range from the vacuum ultraviolet to the soft x-ray region [1,2]. The high order harmonics which are emitted in a series of attosecond bursts with high spatial and temporal coherence are very interesting for applications in physics, chemistry, and biology [3]. The phase matching between the fundamental laser field and the harmonic field, i.e., the coherent construction of the harmonic field in the macroscopic medium, plays a very important role in HHG. In general, the total phase mismatch may be expressed as [4]

$$
\Delta \mathrm{k}_{\mathrm{q}}=2 \pi \mathrm{qp}(1-\eta) \delta \mathrm{n} / \lambda-\mathrm{pq \eta N}_{\mathrm{at}} \mathrm{r}_{\mathrm{e}} \lambda+(\text { geometric term })+(\text { dipole phase term })
$$

where $\lambda$ is the laser wavelength; $p$ and $\eta$ are the gas pressure and ionization fraction, respectively; $\delta \mathrm{n}=\mathrm{n}_{\text {laser }}-\mathrm{n}_{\mathrm{q}} ; \mathrm{n}_{\text {laser }}$ and $\mathrm{n}_{\mathrm{q}}$ are the refractive indices for the laser light and the $\mathrm{q}^{\text {th }}$ harmonic, respectively; and $\mathrm{N}_{\mathrm{at}}$ is the atom density.

In Eq. (1) for the phase mismatch the first (positive) term is related to the medium dispersion and the second (negative) term is due to the plasma dispersion. In principle, phase matching in HHG can be achieved by balancing the different phase mismatch terms in Eq.(1) to obtain a zero total phase mismatch. The generation of phase matched HHG in the near cut-off region by using a moveable focusing lens in a semi-infinite gas cell has been obtained [5]. Phase matched high harmonic generation at very high photon energy is difficult to achieve because the required strong driving field creates a large free electron dispersion through strong ionization of the gas and as a result phase matching cannot be achieved by simply varying the pressure. Thus, extension of the cut-off energy to shorter wavelengths is still one of the major challenges in HHG experiments.

In this paper we propose a new experimental approach in which an off-axis laser beam is used to control the phase matching condition in HHG in a semi-infinite gas cell. Through controlling the phase matching condition the harmonic emission can be suppressed or enhanced. A high photon energy up to $220 \mathrm{eV}(\sim 5.5 \mathrm{~nm}$ in wavelength) from helium gas is observed in our experiments when the off-axis beam is applied. We also demonstrate the important role of the dipole phase on the phase matched HHG process which has not been included in most previous studies.

This is an Open Access article distributed under the terms of the Creative Commons Attribution License 2.0, which permits unrestricted use, distribution, and reproduction in any medium, provided the original work is properly cited. 


\section{Experiments and Discussion}

A $1 \mathrm{kHz}$ multi-stage, multi-pass, chirped-pulse amplifier system which produces $2.5 \mathrm{~mJ}$ pulses with a duration of $30 \mathrm{fs}$ and centred at $810 \mathrm{~nm}$ is used in our experiment. Details of the HHG setup and the detection system have been described elsewhere [5]. The laser beam is split into two beams with an intensity ratio of about 1:4. The two laser beams are focused to a single spot in a gas-filled cell at an angle of about $5^{\circ}$. The first beam with low intensity is used as the off-axis beam and the second beam is used for generation of the harmonics. To produce a time delay between the two beams, a step motor with a spatial resolution of $25 \mathrm{~nm}$ is installed. A positive time delay means that the first beam precedes the second beam. Argon and helium gases are used in this study. When the intensity of the second beam is $>6 \times 10^{14} \mathrm{~W} / \mathrm{cm}^{2}$ enhancement of the ionization and Rayleigh scattering from free electrons in the ovelapping region can be employed for spatial and temporal alignment of the pulses. In this configuration, the total interaction medium is divided into two regions: the first region without any influence of the off-axis beam and the second region (overlapping region) with strong influence of the off-axis beam. In addition, the length of the overlapping region is only a fraction of the total interaction length.

Firstly, we show the possibility of using the off-axis beam to turn off the harmonic emission. In this experiment, the delay of the off-axis beam is set to a very long delay (e.g., $\sim 1 \mathrm{ps}$ ) so that there is not any influence of the off-axis beam on the second beam. Under these conditions, the generation of harmonics is optimized for phase matching along the axis of the second pulse in the observed spectral range. Then the harmonic intensity is scanned following the delay between two beams. Figure 1a shows the intensity of the harmonic $\mathrm{H} 23$ from argon versus the time delay when the intensity of the first pulse is $\sim 10^{13} \mathrm{~W} / \mathrm{cm}^{2}$. A similar dependence is observed for other harmonics ranging from $\mathrm{H} 17$ to $\mathrm{H} 27$ and also for the harmonic spectrum from helium. It is clear that when the polarizations of the two laser fields are parallel, the harmonic signal is dramatically disrupted around zero delay time. This phenomenon is due to the off-axis beam leading to a variation of the phase mismatch in the overlapping region. The change of the phase mismatch results in a change of the phase of the harmonic field in this region. Thus, the relative phase of the harmonic field in the second region compared to that of the harmonic field in the first region can be controlled. At a particular point, these two harmonic fields are strongly subtracted from each other and hence the phase matched harmonic emission established by optimization of the second laser field is now disrupted, i.e., the harmonic signal is turned off. When the polarizations of the two laser fields are crossed the change in the harmonic intensity is small. Since the ionization rate of the atomic medium in the overlapping region under the influence of the first pulse should be independent of the polarization, the contribution of plasma dispersion (the second term of equation 1) to the change of the total phase mismatch does not need to be considered. In addition, for a weak off-axis beam, the instantaneous response of the medium involving the nonlinear refractive index which follows the intensity profile of the fundamental laser field can be ignored. This means that the variation of the medium dispersion phase mismatch (the first term of equation 1) in the presence of the off-axis beam is also negligible. Therefore, the change of the total phase mismatch in the overlapping region will be caused mainly by the dipole phase. The interaction of the second field with the free electrons influences the trajectory and re-scattering of the electrons causes a large change in the dipole phase. Additional evidence for the dominant contribution of the harmonic dipole phase to suppression of the harmonic emission is the different response of the harmonic intensity on the positive and negative sides close to zero time delay (Fig. 1a). For negative delay, the generating beam precedes the off-axis beam, so that when the interaction between two beams occurs, the electrons have already been in the continuum state. Conversely, for positive delay, this interaction occurs when the electrons are still in the ground state leading to a slower response.

When a more intense first laser field $\left(\sim 3 \times 10^{13} \mathrm{~W} / \mathrm{cm}^{2}\right)$ is applied, as shown in Fig. 1b, the temporal profile of the turn-off region is much broader than the pulse duration for the case of parallel fields because the response of the free electrons is very sensitive to the external field and therefore the small field in the tail of the first pulse can disturb the trajectory of the free electrons. Furthermore, for a high intensity first field, the contribution of the instantaneous response of the nonlinear 
refractive index needs to be considered and the variation of the HHG intensity for crossed polarizations is similar to the intensity profile of the fundamental laser field.

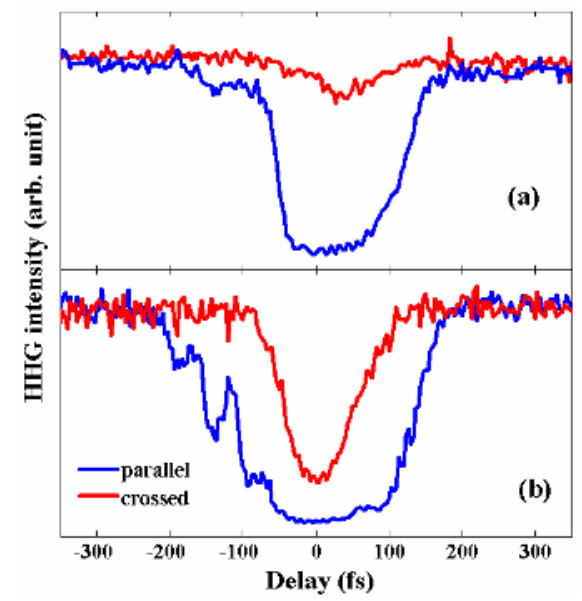

Fig. 1 Variation of the intensity of harmonic H23 from argon gas versus delay between the two laser pulses. The intensity of the off-axis beam is (a) $1 \times 10^{13} \mathrm{~W} / \mathrm{cm}^{2}$ and (b) $3 \times 10^{13} \mathrm{~W} / \mathrm{cm}^{2}$

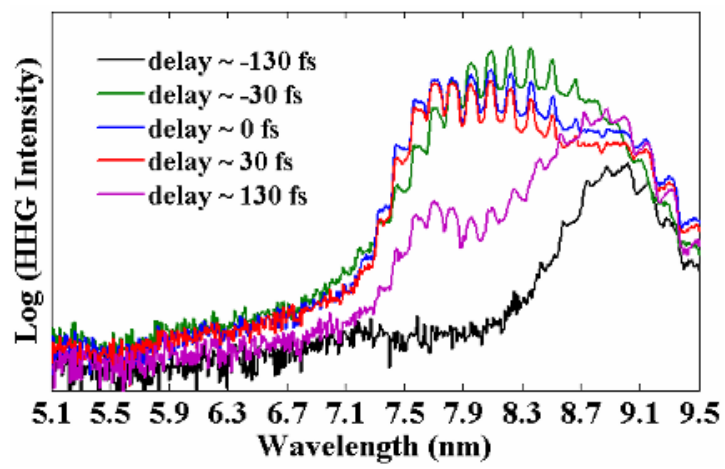

Fig. 2 HHG spectra plotted on a log scale from helium gas with different time delays between the two beams

The possibility of using an off-axis beam to enhance phase matching leading to improvement of harmonic emission is shown in Fig. 2 which plots on a log scale HHG spectra from helium gas with different time delays between the two beams. The same enhancement effect is seen for HHG from argon gas but the generation of very high harmonic orders from helium with high laser intensity is more interesting. The influence of the off-axis beam is strong for delay times of 0 and $\pm 30 \mathrm{fs}$ when the intensity of the off-axis field is high and a large variation of the dipole phase is expected. Thus, the harmonic field in the second region is varied and is added to the harmonic field in the first region. Consequently, the overall harmonic field at the exit of the medium can be strongly enhanced. With an off-axis beam harmonics can be generated down to $7.3 \mathrm{~nm}$ and a second cut-off, with low flux, occurs down to $\sim 5.5 \mathrm{~nm}$. Meanwhile it has been shown that without an off-axis beam the shortest harmonic wavelength that can be obtained is $\sim 8.3 \mathrm{~nm}$ in a semi-infinite gas cell [6].

\section{Conclusion}

We have demonstrated the use of an off-axis laser beam to alter the phase mismatch leading to suppression or enhancement of the HHG process. With an off-axis beam harmonics around $7.3 \mathrm{~nm}$ have been generated from helium gas and a harmonic signal with low flux down to $5.5 \mathrm{~nm}$ has been observed. Additionally, through this study, the importance of the harmonic dipole phase on the phase mismatch has been investigated.

\section{References}

1. J. J. Macklin, J. D. Kmetec, C. L. Gordon, III, Phys. Rev. Lett., 70, 766 (1993).

2. J. Zhou, J. Peatross, M. M. Murnane, H. C. Kapteyn, Phys. Rev. Lett., 76, 752 (1996).

3. A. Rundquist, C. G. Durfee III, Z. Chang, C. Herne, S. Backus, M. M. Murnane, H. C. Kapteyn, Science, 280, 1412 (1998).

4. T. Pfeifer, C. Spielmann, G. Gerber, Rep. Prog. Phys. 69, 443 (2006).

5. L. V. Dao, S. Teichmann, J. Davis, P. Hannaford, J. Appl. Phys. 104, 023105 (2008).

6. S. Teichmann, P. Hannaford, L. V. Dao, Appl. Phys. Lett., 94, 171111 (2009). 